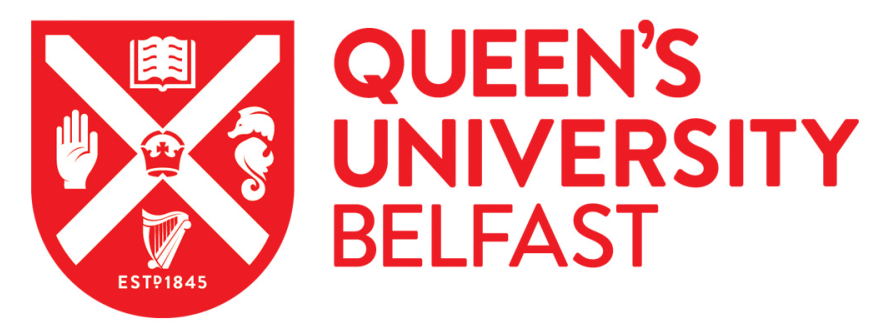

\title{
Molecular Arrangement and Charge Transfer in C60/Graphene Heterostructures
}

Ojeda-Aristizabal, C., Santos, E. J. G., Onishi, S., Yan, A., Rasool, H. I., Kahn, S., Lv, Y., Latzke, D., Velasco, J. J., Crommie, M. F., Sorensen, M., Gotlieb, K., Lin, C-Y., Watanabe, K., Taniguchi, T., Lanzara, A., \& Zettl, A. (2017). Molecular Arrangement and Charge Transfer in C60/Graphene Heterostructures. ACS Nano. https://doi.org/10.1021/acsnano.7b00551

Published in:

ACS Nano

Document Version:

Peer reviewed version

Queen's University Belfast - Research Portal:

Link to publication record in Queen's University Belfast Research Portal

Publisher rights

Copyright () 2017 American Chemical Society.

This work is made available online in accordance with the publisher's policies. Please refer to any applicable terms of use of the publisher.

\section{General rights}

Copyright for the publications made accessible via the Queen's University Belfast Research Portal is retained by the author(s) and / or other copyright owners and it is a condition of accessing these publications that users recognise and abide by the legal requirements associated with these rights.

Take down policy

The Research Portal is Queen's institutional repository that provides access to Queen's research output. Every effort has been made to ensure that content in the Research Portal does not infringe any person's rights, or applicable UK laws. If you discover content in the Research Portal that you believe breaches copyright or violates any law, please contact openaccess@qub.ac.uk. 


\section{Article}

\section{Molecular Arrangement and Charge Transfer in C/Graphene Heterostructures}

Claudia Ojeda-Aristizabal, Elton J. G. Santos, Seita Onishi, Aiming Yan, Haider Imad Rasool, Salman

Kahn, Yinchuan Lv, Drew Latzke, Jairo Velasco Jr., Michael F. Crommie, Matthew Sorensen, Kenneth

Gotlieb, Chiu-Yun Lin, Kenji Watanabe, Takashi Taniguchi, Alessandra Lanzara, and Alex Zettl

ACS Nano, Just Accepted Manuscript • DOI: 10.1021/acsnano.7b00551 • Publication Date (Web): 24 Apr 2017

Downloaded from http://pubs.acs.org on May 3, 2017

\section{Just Accepted}

"Just Accepted" manuscripts have been peer-reviewed and accepted for publication. They are posted online prior to technical editing, formatting for publication and author proofing. The American Chemical Society provides "Just Accepted" as a free service to the research community to expedite the dissemination of scientific material as soon as possible after acceptance. "Just Accepted" manuscripts appear in full in PDF format accompanied by an HTML abstract. "Just Accepted" manuscripts have been fully peer reviewed, but should not be considered the official version of record. They are accessible to all readers and citable by the Digital Object Identifier (DOI@). "Just Accepted" is an optional service offered to authors. Therefore, the "Just Accepted" Web site may not include all articles that will be published in the journal. After a manuscript is technically edited and formatted, it will be removed from the "Just Accepted" Web site and published as an ASAP article. Note that technical editing may introduce minor changes to the manuscript text and/or graphics which could affect content, and all legal disclaimers and ethical guidelines that apply to the journal pertain. ACS cannot be held responsible for errors or consequences arising from the use of information contained in these "Just Accepted" manuscripts. 
SCHOLARONE $^{\text {w }}$
Manuscripts 


\title{
Molecular Arrangement and Charge Transfer in $\mathrm{C}_{60} /$ Graphene Heterostructures
}

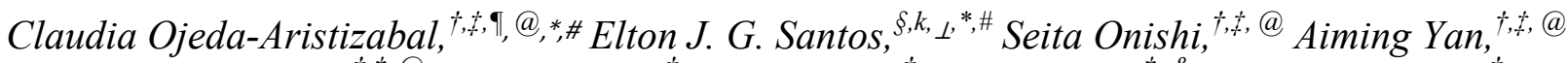 \\ Haider I.Rasool, ${ }^{\dagger,+} @$ Salman Kahn, ${ }^{\dagger}$ Yinchuan Lv, ${ }^{\dagger}$ Drew Latzke, ${ }^{+,},{ }^{\star}$ Jairo Velasco Jr., ${ }^{\dagger}$ \\ Michael F.Crommie, ${ }^{\dagger,+} @$ Matthew Sorensen, ${ }^{\dagger}$ Kenneth Gotlieb, ${ }^{+, \&}$ Chiu-Yun Lin, ${ }^{\dagger}$ Kenji \\ Watanabe, ${ }^{*}$ Takashi \\ Taniguchi, ${ }^{*}$ Alessandra Lanzara, ${ }^{+, \dot{\dagger}}$ and Alex Zettl ${ }^{\dagger, t} @, \#$ \\ ${ }^{\dagger}$ Department of Physics, University of California, Berkeley, CA 94720, USA \\ ${ }^{\ddagger}$ Materials Sciences Division, Lawrence Berkeley National Laboratory, Berkeley, CA 94720 , \\ USA \\ ${ }^{\&}$ Applied Science and Technology, University of California, Berkeley, CA 94720, USA \\ ${ }^{\S}$ Department of Chemical Engineering, Stanford University, Stanford, CA, 94305, USA \\ ${ }^{¥}$ Advanced Materials Laboratory, National Institute for Materials Science, 1-1 Namiki \\ Tsukuba 305-0044, Japan \\ ${ }^{\circledR}$ Kavli Energy NanoSciences Institute at the University of California, Berkeley and the \\ Lawrence Berkeley National Laboratory, Berkeley, CA 94720, USA \\ * These authors contribute equally to this work.
}

\begin{abstract}
Charge transfer at the interface between dissimilar materials is at the heart of electronics and photovoltaics. Here we study the molecular orientation, electronic structure, and local charge transfer at the interface region of $\mathrm{C}_{60}$ deposited on graphene, with and without supporting substrates such as hexagonal boron nitride. We employ ab initio density functional theory with van der Waals (vdW) interactions, and experimentally characterize interface devices using high-
\end{abstract}


resolution transmission electron microscopy and electronic transport. Charge transfer between $\mathrm{C}_{60}$ and the graphene is found to be sensitive to the nature of the underlying supporting substrate and to the crystallinity and local orientation of the $\mathrm{C}_{60}$. Even at room temperature, $\mathrm{C}_{60}$ molecules interfaced to graphene are orientationally locked into position. High electron and hole mobilities are preserved in graphene with crystalline $\mathrm{C}_{60}$ overlayers, which has ramifications for organic high-mobility field-effect devices.

KEYWORDS: graphene, $\mathrm{C}_{60}$, organic semiconducting molecules, fullerenes, interfacial charge transfer

$\mathrm{C}_{60}$ and graphene are, in their own right, fascinating low dimensional materials with spectacular properties. The high electron affinity and unusual phonon modes of $\mathrm{C}_{60}$ have rendered it an important nanomaterial for electronic applications, and the ultrafast charge transfer dynamics and an energetically favorable LUMO level for many donor organic semiconductors have established $\mathrm{C}_{60}$ as an excellent electron acceptor for organic solar cells. ${ }^{1,2}$ When combined with strong charge transfer from alkali metals, the phonon modes in $\mathrm{C}_{60}$ drive superconductivity at surprisingly high temperature. ${ }^{3}$ The related carbon allotrope graphene displays phenomenal electron and hole mobilities even at room temperature, and the conductance can be readily gated by an external electric field or altered via adsorbed chemical species. It is likely that many applications of graphene will involve heterostructures. Indeed, the interfacing of graphene to other low-2D materials via van der Waals forces is a rapidly expanding field of research.,6,20

The interfacing of $\mathrm{C}_{60}$ and graphene is of particular interest. For example, charge transfer between $\mathrm{C}_{60}$ and graphene affects the graphene electronic transport. ${ }^{27-30}$ Adding gating electric fields ${ }^{7,8}$ allows further functional possibilities on the control of interface properties such as 
energy barriers, magnetism ${ }^{9,10}$ and electron-hole dynamics. ${ }^{11,4}$ The ability to fine-tune the charge transfer state of $\mathrm{C}_{60}$ interfaced to graphene (e.g. with reversible electrostatic gating) is thus central to the development of novel electrochemical and optoelectronic prototypes. Devices based on organic molecule/graphene interfaces, which utilize the tunable Fermi level in graphene, have already shown promise. ${ }^{12-14}$ In particular, the $\mathrm{C}_{60}$ /graphene heterostructure is known to form a Schottky junction. ${ }^{13}$

In this report we investigate the $\mathrm{C}_{60}$ /graphene interface via ab initio density functional theory (DFT) calculations, transmission electron microscopy, and electronic transport experiments, at cryogenic temperature and at room temperature. The graphene is either suspended or supported on different substrates, including hexagonal boron nitride (h-BN). We determine the most favorable molecular orientations of the $\mathrm{C}_{60}$ s, the interface band structure, the intrinsic charge transfer (which is highly dependent on $\mathrm{C}_{60}$ deposition process and substrate type), and the graphene electron and hole mobilities. The theoretical and experimental evidence suggests that $\mathrm{C}_{60}$ molecules at the interface remain firmly locked to the graphene lattice even at room temperature, in sharp contrast to the freely rotating behavior of $\mathrm{C}_{60} \mathrm{~S}$ in undoped molecular crystals.

\section{Results and discussion:}

We first examine theoretically the most favorable geometrical arrangements of crystalline close-packed $\mathrm{C}_{60}$ on graphene on different substrates at low temperatures, and the associated interfacial band structure and charge transfer. Figs. 1a,b show schematic representations respectively of $\mathrm{C}_{60}$ on suspended graphene, and $\mathrm{C}_{60}$ on graphene supported by h-BN. The $\mathrm{C}_{60}$ film is assumed to be crystalline with a well-ordered periodic lattice and a $4 \times 4$ superstructure is defined. In the nomenclature of ref. 13 , the $\mathrm{C}_{60}$ lattice is oriented $28^{\circ}$ with respect to the 
graphene lattice, i.e. it is aligned with the armchair direction. Due to the highly spherical shape of the $\mathrm{C}_{60}$ molecule several possible molecular arrangements for $\mathrm{C}_{60}$ on graphene are possible, with different interaction energies and different local charge transfer.

As in ref. 13, we consider nine representative orientations of $\mathrm{C}_{60}$ on graphene (Fig. 1c). We perform first-principles density functional theory calculations including van der Waals (vdW) dispersion forces (the calculation approach differs somewhat from that of ref. 13; see Methods for details). If vdW interactions are excluded (using the PBE functional approach, see Methods), then the most energetically favorable configuration of $\mathrm{C}_{60}$ on graphene is the Hexagon/Hole alignment. However, if vdW interactions are included (using the DRSLL functional approach), then, as presented in Fig. 1d (open bars), the most energetically favorable configuration is the Hexagon/Bridge alignment (right-hand-most open bar), with an interaction energy of about $1.1 \mathrm{ev} / \mathrm{C}_{60}$. We note that the $\mathrm{vdW}$ term is significant, with a jump in interaction energy of roughly $1 \mathrm{eV} / \mathrm{C}_{60}$, as shown previously. ${ }^{13}$ If an h-BN support is added to the graphene (see Fig. 1b), the interaction energy (including vdW interactions) between graphene and $\mathrm{C}_{60}$ is further enhanced by $\sim 0.25-0.33 \mathrm{eV}$, as seen by comparing the open and filled bars in Fig. $1 \mathrm{~d}$. With an h-BN supporting substrate, the most energetically favorable configuration at low temperatures of $\mathrm{C}_{60}$ on graphene remains Hexagon/Bridge (Fig. 1d, right-hand-most filled bar), with an interaction energy of about $1.45 \mathrm{eV} / \mathrm{C}_{60}$.

Different molecular orientations of $\mathrm{C}_{60}$ on graphene give different magnitudes of charge transfer between the $\mathrm{C}_{60}$ and graphene. We use first principles DFT simulations to evaluate the magnitude and sign of the charge transfer. Even though DFT is known to underestimate band gaps and frontier orbitals (HOMO, LUMO), we will show below that our calculations reproduce closely the magnitudes and trends observed in the experiments for the large systems used in the 
supercell. The system size is too large for higher levels of theory, e.g. GW or hybrid functional, to be employed. We consider first the case of $\mathrm{C}_{60}$ on suspended graphene. Fig. 2a shows the calculated charge transfer from $\mathrm{C}_{60}$ to graphene for the different $\mathrm{C}_{60}$ orientations, as a function of $\mathrm{n}_{\mathrm{o}}$, the externally induced graphene charge density (as might result from external electric field gating). With zero external gating $\left(\mathrm{n}_{\mathrm{o}}=0\right)$, the charge transfer is negative in the range 0.03-0.07 electrons $/ \mathrm{C}_{60}$ (this corresponds to a change in charge density in the graphene of 4.3$\left.10.1 \times 10^{12} / \mathrm{cm}^{2}\right)$. For all molecular orientations, with no external gating, $\mathrm{C}_{60}$ on suspended graphene acts as an electron acceptor, as expected. With increasing $\mathrm{n}_{\mathrm{o}}$ in the negative direction, the charge transfer between $\mathrm{C}_{60}$ and graphene increases, with the Dimer/Hole orientation giving a slightly bigger effect. With increasing $\mathrm{n}_{\mathrm{o}}$ in the positive direction, external gating compensates the intrinsic charge transfer, with a zero crossing at about $\mathrm{n}_{\mathrm{o}}=+20 \times 10^{12} / \mathrm{cm}^{2}$. Beyond this, external gating overcomes the intrinsic charge flow and the $\mathrm{C}_{60}$ is forced to donate electrons to the graphene.

Figs. $2 \mathrm{~b}$ and $2 \mathrm{c}$ show respectively the influence of external gating (again expressed as $\mathrm{n}_{\mathrm{o}}$ ) on the graphene electronic density of states and Fermi level shift, for the $\mathrm{C}_{60}$ /graphene hybrid system assuming a Dimer/Hole configuration. Similar results are obtained for the other orientations. The effect of electrostatic gating on the electronic band structure is displayed in Figs. 2d-f, which clearly show the charge doping effect at the Dirac point and the rigid shift-like effect of the electric gating on $\mathrm{C}_{60}$ and graphene bands. Once the $\mathrm{C}_{60}$ /graphene interface is created, at no bias regime $n_{0}=0$, the HOMO of the $C_{60}$ layer (faint red in Fig. 2e) is sufficiently lower in energy relative to the Fermi level and charge transfer takes place from graphene to $\mathrm{C}_{60}$. At finite bias (Fig. 2d, 2f), the potential difference between graphene and $\mathrm{C}_{60}$ drives the position of the fullerene states closer (for $\mathrm{n}_{\mathrm{o}}<0$ ) or away (for $\mathrm{n}_{\mathrm{o}}>0$ ) from the Fermi level by several tenths 
of meV's. This particular arrangement of graphene and $\mathrm{C}_{60}$ states drives the system to a band alignment where most of the states are bias-dependent allowing further control on the electronic structure.

Figs. $3 \mathrm{a}$ and $3 \mathrm{~b}$ contrast, for $\mathrm{C}_{60} /$ graphene with $\mathrm{n}_{\mathrm{o}}=0$, the charge transfer and induced dipole moment $\mid \mathrm{P}($ Debye $) \mid$ for the selected $\mathrm{C}_{60}$ orientations. The induced dipole moments are all in the range $\sim 0.3-0.5$ Debye, with no obvious correlation to the induced charge densities. For $\mathrm{C}_{60} / \mathrm{G}$, the charge transfer increases with increasing magnitude of dipole moment but there is much scatter in the data (see Fig. S5a). There appears to be no correlation between the $\mathrm{C}_{60}$ adsorption orientation dependence of the dipole moment, charge transfer and interaction energies. Fig. 3c shows the zero bias charge difference density plot for the Dimer/Hole configuration for the $\mathrm{C}_{60}$ /graphene heterostructure.

We now turn to the additional influence of a supporting h-BN substrate on the charge transfer from $\mathrm{C}_{60}$ to graphene. Fig. $3 \mathrm{~d}$ shows the predicted charge transfer from $\mathrm{C}_{60}$ to graphene for the different molecular orientations of $\mathrm{C}_{60}$, all for the bias-free condition $\mathrm{n}_{\mathrm{o}}=0$. In all cases the charge transfer is negative (electrons again flow from graphene to $\mathrm{C}_{60}$ ), and the Dimer/Hole orientation has the maximum charge transfer. The orientation with the maximum charge transfer (Dimer/Hole) is different from the orientation with the strongest interaction energy (Hexagon/Bridge), which implies that more charge transfer does not necessarily cause stronger molecular interaction. The interaction of $\mathrm{C}_{60}$ with the substrate is an interplay between vdW interaction at the $\mathrm{C}_{60}$ /graphene interface and charge rearrangement at the graphene/h-BN interface. Hence, it is possible that the vdW interaction is not determined solely by charge transfer. As the spread in interaction energies among the $\mathrm{C}_{60}$ molecular orientations is small 
(0.020-0.100 eV), it is possible to establish more than one $\mathrm{C}_{60}$ molecular orientation. Due to this complication, further study is needed to exactly determine the tradeoff between the driving forces in this system. Remarkably, the addition of the h-BN substrate reduces the maximum (bias-free) charge transfer by roughly one order of magnitude from that of the $\mathrm{C}_{60} /$ graphene configuration (Fig. 3a). Fig. 3e shows the corresponding induced dipole moment $\mid P($ Debye $) \mid$. Interestingly, with an h-BN substrate present, the polarizations for the nine molecular arrangements can be separated into two groups, delineated by a threshold polarization $\mid P($ Debye $) \mid$ thresh $=0.10$, as indicated by the dotted line in Fig. 3e. Results for the group above threshold are displayed with blue bars and give sizeable charge transfer in the range of $0.01-0.28 \times 10^{12} / \mathrm{cm}^{2}$ $\left(0.07-2 \times 10^{-3} \mathrm{e} / \mathrm{C}_{60}\right)$. Results of the group below threshold are displayed in purple and give charge transfers 3 orders of magnitude lower. For $\mathrm{C}_{60} / \mathrm{G} / \mathrm{h}-\mathrm{BN}$, there is no clear correlation between charge transfer and dipole moment (see Fig. S5b). The only notable qualitative trend is that the group of molecular orientations with large dipole moments corresponds to the group with large charge transfer and the same goes for the group of small dipole moments and charge transfer. The greatest charge transfer is obtained from the Dimer/Hole configuration $\left(1.8 \times 10^{-3} \mathrm{e} / \mathrm{C}_{60}\right)$. The charge transfer varies significantly across the $\mathrm{C}_{60}$ configurations due to the importance of the carbon bond between two hexagons of the $\mathrm{C}_{60}$ cage, namely 6:6. According to the chemistry of $\mathrm{C}_{60}{ }^{16,17} 6: 6$ bonds are preferential sites for functionalization for several compounds as the degree of electron delocalization varies (double bond) and higher reactivity is present, mainly due to the increase of $\pi$ electron density at this bond.

Although h-BN is often considered to be an ideal, inert substrate for graphene, ${ }^{18}$ our results clearly show that it has a profound effect on heterostructure charge transfer and polarizability. Fig. $3 f$ presents the charge difference density plot at zero bias for the Dimer/Hole configuration 
for $\mathrm{C}_{60} /$ graphene/h-BN. The stronger charge transfer for $\mathrm{C}_{60}$ on the suspended graphene with no underlying h-BN (Fig. 3c) is strikingly evident. Prior to forming the interface with $\mathrm{C}_{60}$, the induced dipole moment at the graphene/h-BN heterostructure is $\mid P($ Debye $) \mid \approx 0.33$ (see Figure S4). After forming the interface with $\mathrm{C}_{60}$, charge rearrangement takes place and the value reduces to below $\mid P($ Debye $) \mid \approx 0.10$. The difference in electronic character for the $p_{z}$-orbitals between $\mathrm{h}-\mathrm{BN}$, polar, and graphene, covalent, is one of the main driving-forces for such modifications of the interfacial polarization dipole. No appreciable modifications $(<0.10 \AA)$ of the value of the interlayer distance between graphene and $\mathrm{C}_{60}$ are observed due to this charge rearrangement once monolayer or few-layers h-BN are introduced. This suggests the active electronic role of the h$\mathrm{BN}$ substrate in limiting the amount of carriers that participates in the interfacial charge separation.

We note that the theoretical studies described above are performed in the low temperature $(\mathrm{T}=0)$ regime. The question arises what influence higher temperature might have on the results. For example, it is well known that in (undoped) crystalline $\mathrm{C}_{60}$, the $\mathrm{C}_{60}$ molecules freely rotate at room temperature. ${ }^{1,2}$ Near $260 \mathrm{~K}$ the $\mathrm{C}_{60}$ s rotate almost freely, and in the range $260 \mathrm{~K}$ to $90 \mathrm{~K}$ they execute jumps. Below $90 \mathrm{~K}$, the $\mathrm{C}_{60}$ s are substantially frozen in place. ${ }^{1}$ On the other hand, for alkali-doped $\mathrm{C}_{60}$, the situation is quite different. Here the $\mathrm{C}_{60} \mathrm{~s}$ are locked into specific orientations with respect to the alkali ions, even at room temperature. X-ray diffraction studies on $\mathrm{K}_{3} \mathrm{C}_{60}$ clearly find the absence of $\mathrm{C}_{60}$ free rotations. ${ }^{3}$ For $\mathrm{K}_{3} \mathrm{C}_{60}$ and $\mathrm{Rb}_{3} \mathrm{C}_{60}$, the $\mathrm{C}_{60} \mathrm{~s}$ orient themselves so that the electron-rich $\mathrm{C}=\mathrm{C}$ bonds face the $\mathrm{K}^{+}$and $\mathrm{Rb}^{+}$ions for charge transfer. Combined with effects of molecular repulsion, $\mathrm{C}_{60}$ molecules are fixed to one of the two energetically stable orientations and switch between the two with thermal energy. ${ }^{1,2}$ For ions with smaller ionic radii, such as $\mathrm{Li}^{+}$and $\mathrm{Na}^{+}$, the ion- $\mathrm{C}_{60}$ repulsion is much weaker, but the $\mathrm{C}_{60} \mathrm{~S}$ 
are still locked to a few energetically stable orientations and jump between them with thermal energy. $^{1,2,5}$

We have examined theoretically the situation for $\mathrm{C}_{60}$ on graphene where the pure $\mathrm{C}_{60}$ becomes "doped" (i.e. charged) by virtue of interaction with the (possibly gated) graphene. In this case the $\mathrm{C}_{60} \mathrm{~S}$ that reside at the interface with graphene are substantially locked into position even at room temperature as demonstrated using ab initio molecular dynamics simulations at $300 \mathrm{~K}$ (see SI and movies for details). Hence, the charge transfer and band structure calculations presented above are relevant also for finite temperature. Theoretical simulations predict no temperature dependence of charge transfer up to $300 \mathrm{~K}$.

We now turn to experiment. We explore the following heterostructure: multilayer crystalline $\mathrm{C}_{60}$ on monolayer graphene on multilayer h-BN. Fig. $4 \mathrm{c}$ shows schematically a crosssection of our typical device-like structure, here assuming crystalline $\mathrm{C}_{60}$ and an h-BN support. Fig. 4d shows an optical image of a functioning device.

We have found that the crystallinity of the $\mathrm{C}_{60}$, and the associated device electronic characteristics, are highly sensitive to $\mathrm{C}_{60}$ deposition conditions. If the $\mathrm{C}_{60}$ is evaporationdeposited under only modest $\left(1 \times 10^{-4}\right.$ torr) "uncontrolled" vacuum conditions, an amorphous $\mathrm{C}_{60}$ film typically results (see Fig. S1). On the other hand, if the $\mathrm{C}_{60}$ deposition is performed under more controlled UHV conditions, highly crystalline $\mathrm{C}_{60}$ films are readily obtained. Figs. 4a,b exemplify the high degree of $\mathrm{C}_{60}$ crystallinity of our UHV-prepared samples. For these TEMcompatible samples, $15 \mathrm{~nm}$ of $\mathrm{C}_{60}$ is deposited on suspended graphene at $1.4 \times 10^{-10}$ torr. Excellent crystallinity is observed with a grain size ranging from $\approx 100 \mathrm{~nm}$ to $200 \mathrm{~nm}$, where the projected lattice images of the stacked layers of $\mathrm{C}_{60}$ indicate a triangular pattern for the organization of the molecules (inset in Fig. 4a). The crystalline structure is confirmed with electron diffraction as 
shown in Fig. 4b. The outermost spots (blue circles) correspond to the Bragg reflections for the lattice plane (0-110) of graphene (with $2.1 \AA$-spacing). ${ }^{15}$ The inner spots (red circles) correspond to Bragg reflections for the lattice plane (111) of $\mathrm{C}_{60}$ (with $8.73 \AA$-spacing), which correspond to a close packed plane with a 2D triangular lattice. This result is consistent with previous results for $\mathrm{C}_{60}$ thin films ${ }^{13}$. Substrate heating is crucial for achieving highly crystalline $\mathrm{C}_{60}$ films ${ }^{13}$ (see Methods).

A controlled deposition of the crystalline $\mathrm{C}_{60}$ thin film is essential for quantifying the charge transfer between graphene and $\mathrm{C}_{60}$ (see Fig. S1). Figs. 5a,b show $\mathrm{R}$ vs $\mathrm{V}_{\mathrm{g}}$ data for high quality crystalline $\mathrm{C}_{60}$ /graphene/h-BN heterostructures constructed under UHV conditions $\left(1.4 \times 10^{-10}\right.$ torr), with a $\mathrm{C}_{60}$ thickness of order $50 \mathrm{~nm}$. $\mathrm{C}_{60}$ here behaves as an electron acceptor. The room temperature data of Fig. 5a indicate a charge transfer from graphene to $\mathrm{C}_{60}$ of $0.2 \times 10^{12} / \mathrm{cm}^{2}$, or $\Delta \rho=0.14 \times 10^{-2} \mathrm{e} / \mathrm{C}_{60}$ transferred from graphene to $\mathrm{C}_{60}$. Fig. $5 \mathrm{~b}$ shows similar data for the same device, but for $\mathrm{T}=4.2 \mathrm{~K}$. The charge transfer is again $0.2 \times 10^{12} / \mathrm{cm}^{2}$ or $\Delta \rho=0.14 \times 10^{-2} \mathrm{e} / \mathrm{C}_{60}$, in agreement with $\Delta \rho$ theoretically calculated for the dimer/hole $\mathrm{C}_{60}$ orientation for a $\mathrm{C}_{60} /$ graphene/h-BN heterostructure (see Fig. 3d). As predicted by molecular dynamics simulations, the absence of temperature dependence results in experimental observation of very close charge transfer values at $4.2 \mathrm{~K}$ and $300 \mathrm{~K}$.

The carrier mobility is derived from the resistance plots of Figs. 5a,b. Knowing the aspect ratio of the sample, the measured resistivity is converted to conductivity and using the Drude model $(\sigma=n e \mu)$, the mobility of the sample is deduced. ${ }^{31}$ Simple inspection of the slope of the resistance near the charge neutrality point (Figs. 5a,b) indicates that the high electron and hole mobilities of graphene in the graphene/h-BN devices are largely preserved after $\mathrm{C}_{60}$ deposition. Table 1 shows that both electron and hole mobilities remain within a factor of two after $\mathrm{C}_{60}$ 
deposition. The room temperature $(\mathrm{T}=300 \mathrm{~K})$ data suggest that both the electron and hole mobilities are slightly depressed upon $\mathrm{C}_{60}$ deposition, while the $4.2 \mathrm{~K}$ data suggest a depression in the hole mobility, and an increase in the electron mobility, upon $\mathrm{C}_{60}$ deposition. The increase in electron mobility upon $\mathrm{C}_{60}$ deposition is surprising, and points to a possible modest annealing effect on the graphene during the (elevated temperature) $\mathrm{C}_{60}$ deposition.

\section{Conclusion:}

In summary, $\mathrm{C}_{60} /$ graphene constitutes a van-der-Waals heterostructure with exciting possibilities for electronic devices and charge transfer applications. We have shown through first-principles calculations that charge transfer between $\mathrm{C}_{60}$ and graphene depends on $\mathrm{C}_{60}$ orientation, and, critically, on the presence or absence of an underlying h-BN substrate. The $\mathrm{C}_{60}$ orientation with the strongest interaction energy is Hexagonal/Bridge, but the orientation with the greatest charge transfer and dipole moment is Dimer/Hole. $\mathrm{C}_{60} \mathrm{~S}$ at the graphene interface are orientationally locked into position and do not rotate, even at room temperature. External gating can change the $\mathrm{C}_{60}$ from an electron acceptor to an electron donor. Crystalline $\mathrm{C}_{60} /$ graphene/h$\mathrm{BN}$ devices maintain the high mobility intrinsic to suspended graphene, and have a charge transfer consistent with the Dimer/Hole $\mathrm{C}_{60}$ configuration. Our findings suggest that the combination of $a b$ initio density functional theory calculations and electronic transport experiments can be effective in the search for other substrates that enhance the range of tunable charge transfer at the $\mathrm{C}_{60}$ /graphene interface.

\section{Methods:}




\section{Fabrication of the $\mathrm{hBN}$-graphene- $\mathrm{C}_{60}$ heterostructure and electronic transport}

\section{measurements}

Graphene was mechanically exfoliated from natural graphite on methyl methacrylate (MMA) polymer. Single graphene flakes were selected and transferred on hexagonal boron nitride (h-BN), which was previously deposited by mechanical exfoliation on a $\mathrm{Si} / \mathrm{SiO}_{2}$ wafer. Transfer was done following the procedure described in. ${ }^{20}$ Metallic electrodes were deposited using a silicon shadow mask, ${ }^{21}$ avoiding the use of e-beam resist and further contamination of the surface. After the deposition of the electrodes, the sample was annealed in a $\mathrm{H}_{2} / \mathrm{Ar}$ atmosphere at $350{ }^{\circ} \mathrm{C}$ for three hours in order to clean the surface from any organic contamination. Finally, $\mathrm{C}_{60}$ was deposited in ultrahigh vacuum $\left(\mathrm{P}=1.4 \times 10^{-10}\right.$ torr $)$ using a low temperature effusion cell. The sample was slowly heated up from room temperature to $156{ }^{\circ} \mathrm{C}$ at a rate of $0.5 \mathrm{~K} / \mathrm{min}$. This slow rate protects the integrity of the electrical contact to graphene. Deposition of $\mathrm{C}_{60}$ was done at $0.1 \AA / \mathrm{s}$ heating the effusion cell at $332{ }^{\circ} \mathrm{C}$ and holding the sample temperature at $156{ }^{\circ} \mathrm{C}$ during deposition. Measurements were performed in a vacuum probe station before and after the deposition of $\mathrm{C}_{60}$, at $\mathrm{P}=1 \times 10^{-8}$ Torr at $300 \mathrm{~K}$ and at a lower vacuum at $4.2 \mathrm{~K}$. Two terminal differential resistance measurements were done with a lock-in amplifier applying a small AC current of 10nA. The carrier density was controlled by applying a DC voltage to the doped silicon.

\section{vdW ab initio calculations}

Calculations were based on $a b$ initio density functional theory using the SIESTA method ${ }^{22}$ and the VASP code. ${ }^{23,24}$ Results presented in the manuscript were produced using the VASP code, while SIESTA was used to perform initial tests at large number of atoms in the unit cell. The 


\begin{abstract}
generalized gradient approximation ${ }^{25}$ along with the DRSLL ${ }^{26}$ functional, which includes vdW dispersion forces, were used in both methods, together with a double-polarized basis set in Siesta, and a well-converged plane-wave cutoff of $500 \mathrm{eV}$ in VASP. We used a Fermi-Dirac distribution with an electronic temperature of $\mathrm{k}_{\mathrm{B}} \mathrm{T}=20 \mathrm{meV}$. Additional details are provided in the Supplementary Material.
\end{abstract}

\title{
Corresponding authors:
}

${ }^{\#}$ E-mail: Claudia.Ojeda-Aristizabal@csulb.edu

${ }^{\#}$ E-mail: e.santos@qub.ac.uk

${ }^{\#}$ Email: azettl@berkeley.edu

Present addresses:

IDepartment of Physics and Astronomy, California State University, Long Beach, CA 90840, USA

${ }^{\text {k}}$ School of Mathematics and Physics, Queen's University Belfast, BT7 1NN, UK

${ }^{\perp}$ School of Chemistry and Chemical Engineering, Queen's University Belfast, BT9 5AL, UK

\section{Author's contributions:}

*These authors contributed equally to this work.

\section{Acknowledgments}


CO-A thanks L. Ju for help in sample fabrication and fruitful discussions, and K. Kim for advice on $\mathrm{C}_{60}$ deposition. This work was supported in part by the Director, Office of Science, Basic Energy Sciences, Materials Sciences and Engineering Division of the U.S. Department of Energy under Contract No. DE-AC02-05CH11231 within the sp2-Bonded Materials Program (KC2207) which provided for sample preparation, and within the Molecular Foundry which provided for TEM characterization; and the National Science Foundation under grant DMR1206512 which provided for transport measurements. E.J.G.S. acknowledges the use of computational resources from the UK national high performance computing service, ARCHER, for which access was obtained via the UKCP consortium and funded by EPSRC grant ref EP/K013564/1; and the Extreme Science and Engineering Discovery Environment (XSEDE), supported by NSF grants number TG-DMR120049 and TG-DMR150017. The Queen's Fellow Award through the startup grant number M8407MPH and the Energy Sustainable PRP (QUB) are also acknowledged.

\section{Supplementary Material}

The supplementary material is available free of charge online via the internet at $\mathrm{t}$ http://pubs.acs.org.

\section{References}

(1) Prassides, K.; Margadonna, S. In Fullerenes: Chemistry, Physics and Technology; Kadish, K. M.; Ruoff, R. S., Eds; John Wiley \& Sons Inc., 2000; pp. 249-251, 555-610.

(2) Dresselhaus M. S.; Dresselhaus, G.; Eklund, P. C. In Science of Fullerenes and Carbon Nanotubes; Academic Press Inc, 1996; pp 184-197, 243-246

(3) Stephens, P. W.; Mihaly, L.; Lee, P. L.; Whetten, R. L.; Huang, S.-M.; Kaner, R.; Deiderich, F.; Holczer, K. Structure of Single-Phase Superconducting $\mathrm{K}_{3} \mathrm{C}_{60}$. Nature 1991, $351,632-634$. 
(4) Hebard, A. F.; Rosseinsky, M. J.; Haddon, R. C.; Murphy, D. W.; Glarum, S. H.; Palstra, T. T. M.; Ramirez, A. P.; Kortan, A. R. Superconductivity at 18 K In Potassium-

Doped $\mathrm{C}_{60}$. Nature 1991, 350, 600-601.

(5) Yildirim, T. ; Fischer, J. E.; Harris, A. B.; Stephens, P. W.; Liu, D.; Brard, L.; Strongin, R. M.; Smith, A. B. Orientational Phase Transition in $\mathrm{Na}_{\mathrm{x}} \mathrm{C}_{60}(1<\mathrm{x}<3)$. Phys. Rev. Lett. 1993, 71, 1383-1386.

(6) Geim, A. K.; Grigorieva, I. V. Van der Waals Heterostructures. Nature 2013, 499, 419-425.

(7) Geim, A. K. Graphene: Status and Prospects. Science 2009, 324, 1530-1534.

(8) Geim, A. K.; Novoselov, K. S. The Rise of Graphene. Nat. Mater. 2007, 6, 183191.

(9) Santos, E. J. G. Carrier-Mediated Magnetoelectric Coupling in Functionalized Graphene. ACS Nano 2013, 7, 9927-9932.

(10) Santos, E. J. G. Magnetoelectric Effect in Functionalized Few-Layer Graphene. Phys. Rev. B 2013, 87, 15540-15547.

(11) Li, Z. Q.; Henriksen, E. A.; Jiang, Z.; Hao, Z.; Martin, M. C.; Kim, P.; Stormer, H. L.; Basov, D. N. Dirac Charge Dynamics in Graphene by Infrared Spectroscopy. Nat. Phys. 2008, 4, 532-535.

(12) Ojeda-Aristizabal, C; Bao, W.; Fuhrer, M. S. Thin-Film Barristor: A GateTunable Vertical Graphene-Pentacene Device. Phys. Rev. B 2013, 88, 035435.

(13) Kim, K.; Lee, T. H.; Santos, E. J. G.; Jo, P. S.; Salleo, A.; Nishi, Y.; Bao, Z. Structural and Electrical Investigation of $\mathrm{C}_{60}$-Graphene Vertical Heterostructures. $A C S$ Nano 2015, 9, 5922-5928.

(14) Lee, C. H.; Schiros, T; Santos, E. J. G.; Kim, B.; Yager, K. G.; Kang, S. J.; Lee, S.; Yu, J.; Watanabe, K.; Taniguchi, T.; Hone, J.; Kaxiras, E.; Nuckolls, C.; Kim, P. Epitaxial Growth of Molecular Crystals on Van der Waals Substrates for HighPerformance Organic Electronics. Adv. Mater. 2014, 26, 2812-2817.

(15) Meyer, J. C.; Geim, A. K.; Katsnelson, M. I.; Novoselov, K. S.; Booth, T. J.; Roth, S. The Structure of Suspended Graphene Sheets. Nature 2007, 446, 60-63.

(16) Prato, M.; Lucchini, V.; Maggini, M.; Stimpfl, E.; Scorrano, G.; Eiermann, M.; Suzuki, T.; Wudl, F. Energetic Preference in 5,6 and 6,6 Ring Junction Adducts of $\mathrm{C}_{60}$ : Fulleroids and Methanofullerenes. J. Am. Chem. Soc. 1993, 115, 8479-8480.

(17) Fagan, P.; Calabrese, J. C.; Malone, B. Metal Complexes of Buckminsterfullerene $\left(\mathrm{C}_{60}\right)$. Acc. Chem. Res. 1992, 23, 134-142. 
(18) Dean, C. R.; Young, A. F.; Meric, I.; Lee, C.; Wang, L.; Sorgenfrei, S.; Watanabe, K.; Taniguchi, T.; Kim, P.; Shepard, K. L.; Hone, J. Boron Nitride Substrates for High-Quality Graphene Electronics. Nat. Nanotechnol. 2010, 5, 722-726.

(19) Withers, F.; Del Pozo-Zamudio, O.; Mishchenko, A.; Rooney, A. P.; Gholinia, A.Watanabe, K.; Taniguchi, T.; Haigh, S. J.; Geim, A. K.; Tartakovskii, A. I.; Novoselov, K. S. Light-Emitting Diodes by Band-Structure Engineering in Van der Waals Heterostructures. Nat. Mater. 2015, 14, 301-306.

(20) Zomer, P. J.; Dash, S. P.; Tombros, N.; van Wees, B. J. A Transfer Technique for High Mobility Graphene Devices on Commercially Available Hexagonal Boron Nitride. Appl. Phys. Lett. 2011, 99, 232104-232109.

(21) Bao, W; Liu, G; Zhao, Z; Zhang, H; Yan, D; Deshpande, A.; LeRoy, B.; Lau, C. N. Lithography-Free Fabrication of High Quality Substrate-Supported and Freestanding Graphene Devices. Nano Res. 2010, 3, 98-102.

(22) Soler, Jose M.; Artacho, E.; D Gale, J.; Garcia, A.; Junquera, J,; Orderjon, P.; Sanchez-Portal, D. The SIESTA Method for ab Initio Order-N Materials Simulation. J. Phys.: Condens. Matter 2002 14, 2745-2779.

(23) Kresse, G.; Hafner, J. Ab Initio Molecular Dynamics for Open-Shell Transition Metals. Phys. Rev. B 1993 48, 13115-13118.

(24) Kresse, G.; Furthmller, J. Efficient Iterative Schemes for Ab Initio Total-Energy Calculations Using a Plane-Wave Basis Set. Phys. Rev. B 1996 54, 11169-11186.

(25) Perdew, J. P.; Burke, K.; Ernzerhof, M. Generalized Gradient Approximation Made Simple. Phys. Rev. Lett. 1996, 77, 3865-3868.

(26) Dion, M.; Rydberg, H.; Schroder, E.; Langreth, D. C.; Lundqvist, B. I. Van der Waals Density Functional for General Geometries. Phys. Rev. Lett. 2004, 92, $246401-$ 246405.

(27) Bautista-Flores C., Sato-Berrú R. Y.; Mendoza D. Charge Transfer in the Fullerene $\mathrm{C}_{60}$-Few Layer Graphene System and the Existence of Negative Photoconductivity. Appl. Phys. Lett. 2014, 105, 191116.

(28) Jnawali, G.; Rao, Y.; Beck, J. H.; Petrone, N.; Kymissis, I; Hone, J.; Heinz, T. F. Observation of Ground- and Excited-State Charge Transfer at the $\mathrm{C}_{60}$ /Graphene Interface. ACS Nano 2015, 9, 7175-7185. 
(29) Wang, R; Wang, S.; Wang, X.; Meyer, J. A. S; Hedegard, P.; Laursen, B. W.; Cheng, Z.; Qiu, X. Charge Transfer and Current Fluctuations in Single Layer Graphene Transistors Modified by Self-Assembled $C_{60}$ Adlayers. Small 2013, 9, 2420.

(30) Chaturbedy, P.; Ramakrishna Matte, H.S.S.; Voggu, R.; Govindaraj, A; Rao, C.N.R. Self-Assembly of $\mathrm{C}_{60}$, SWNTs and Few-Layer Graphene and Their Binary Composites at the Organic-Aqueous Interface. J. Colloid Interface Sci. 2011, 360, 249-255.

(31) Novoselov, K.S.; Geim, A.K.; Morozov, N.; Jiang, D.; Zhang Y.; Dubonos S. V.; Grigorieva, I.V.; Firsov, A. A. Electric Field Effect in Atomically Thin Carbon Films. Science 2004, 306, 666-669.

\section{Table 1}

\begin{tabular}{|r|r|r|r|r|r|c|}
\hline & \multicolumn{3}{|c|}{ Before C60 deposition } & \multicolumn{3}{c|}{ After C60 deposition } \\
\hline $\mathrm{T}(\mathrm{K})$ & $\begin{array}{c}\mu_{\mathrm{h}} \\
\left(\mathrm{cm}^{2} / \mathrm{Vs}\right)\end{array}$ & $\begin{array}{c}\mu_{\mathrm{e}} \\
\left(\mathrm{cm}^{2} / \mathrm{Vs}\right)\end{array}$ & $\begin{array}{c}\text { Peak position } \\
\left(\mathrm{cm}^{-2}\right)\end{array}$ & $\begin{array}{c}\mu_{\mathrm{h}} \\
\left(\mathrm{cm}^{2} / \mathrm{Vs}\right)\end{array}$ & $\begin{array}{c}\mu_{\mathrm{e}} \\
\left(\mathrm{cm}^{2} / \mathrm{Vs}\right)\end{array}$ & $\begin{array}{c}\text { Peak position } \\
\left(\mathrm{cm}^{-2}\right)\end{array}$ \\
\hline 4.2 & 12,300 & 5,100 & $-0.086 \pm 0.002$ & $6,150^{*}$ & $10,200^{*}$ & $0.095 \pm 0.002$ \\
\hline 300 & 4,700 & 5,830 & $-0.095 \pm 0.003$ & 2,530 & 4,600 & $0.121 \pm 0.003$ \\
\hline
\end{tabular}

Table 1. Parameters from electronic transport measurements, for a $\mathrm{C}_{60} / \mathrm{G} / \mathrm{h}-\mathrm{BN}$ device. 
Figures

(a)

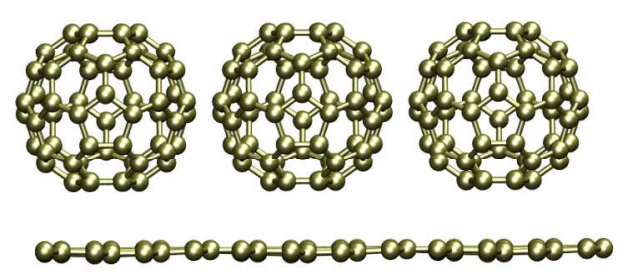

(b)

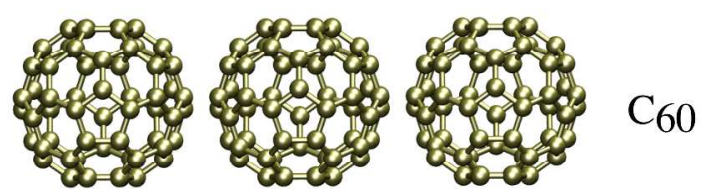
$\infty=\infty=\infty-\infty-\infty=\infty=\infty-\infty=\infty=\infty=\infty$ Graphene $\infty=\infty-\infty=\infty-\infty=\infty-\infty-\infty-\infty=\infty-\infty$ h-BN

(c)
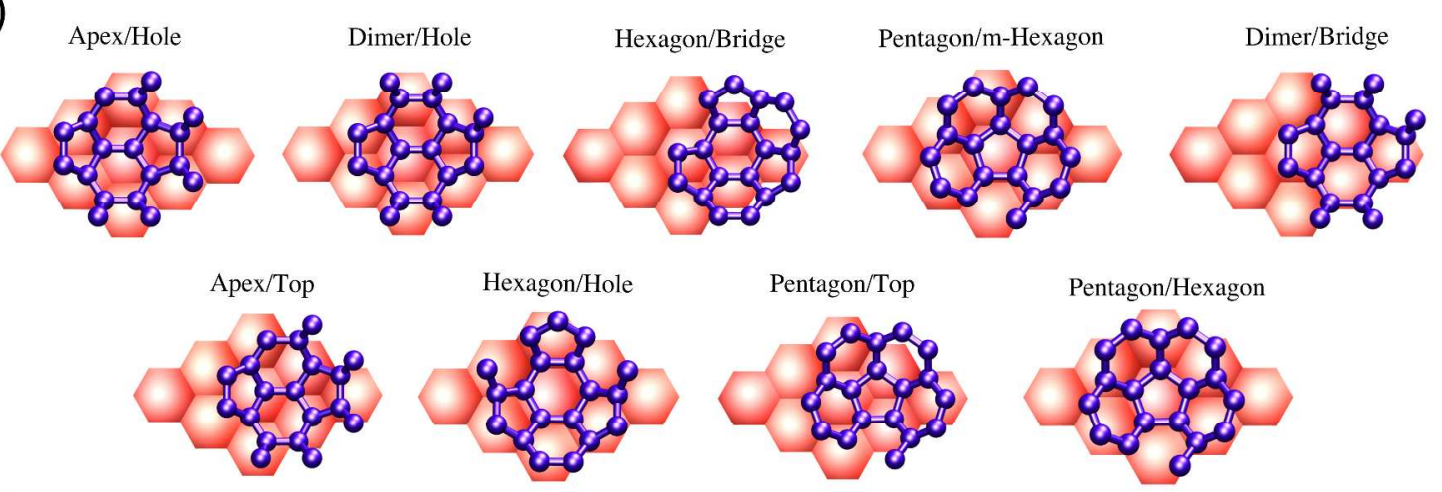

(d)

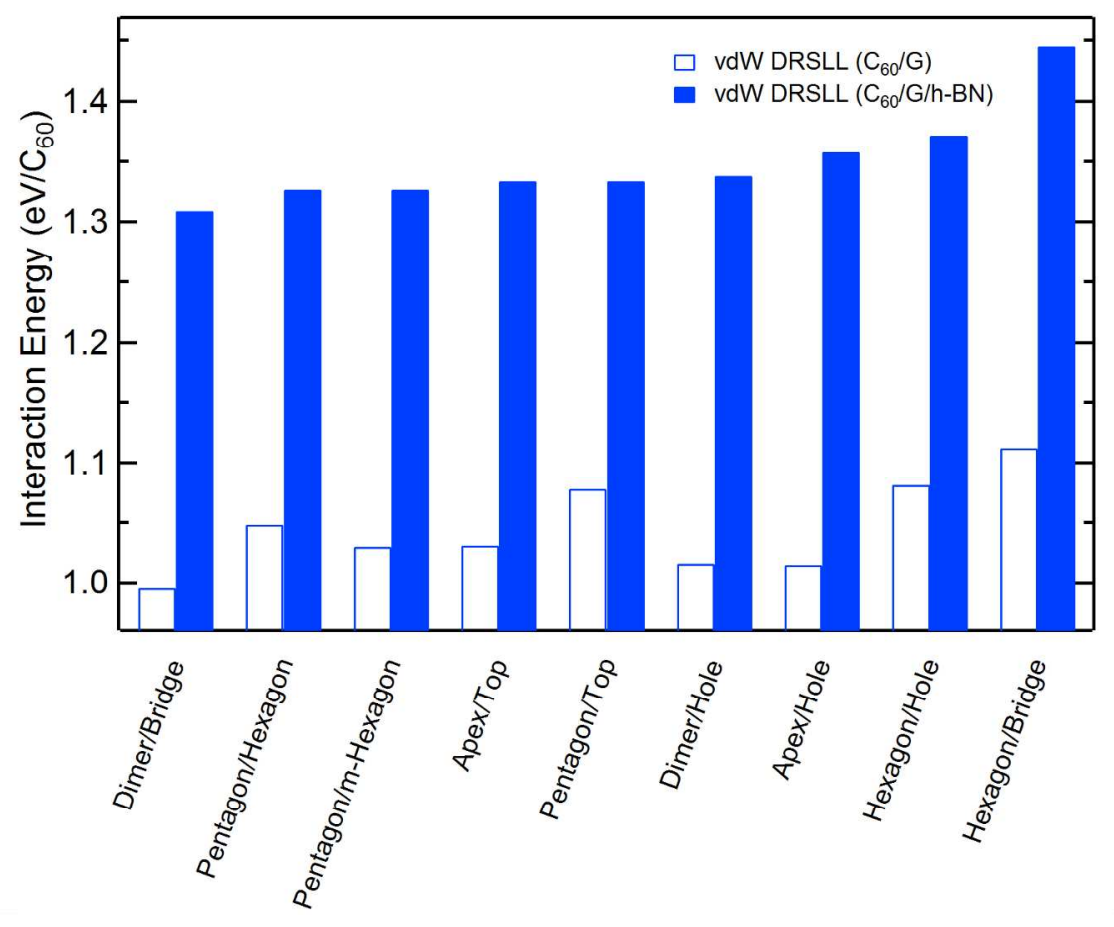


Figure 1. Schematic representation of (a) $C_{60}$ molecules on graphene $\left(C_{60} / G\right)$, and (b) $C_{60}$ molecules on graphene supported by $\mathrm{h}-\mathrm{BN}\left(\mathrm{C}_{60} / \mathrm{G} / \mathrm{h}-\mathrm{BN}\right)$. The relative separation between $\mathrm{C}_{60}$, graphene, and h-BN has been initially converged. (c) Optimized molecular configurations between $\mathrm{C}_{60}$ and graphene resulting from a computational screening at different starting geometries. For clarity, $\mathrm{C}_{60}$ molecules (violet atoms) are only shown near the graphene surface (faint pink) with the rest of the other atoms hidden. (d) Interaction energies per $\mathrm{C}_{60}$ molecule calculated with vdW interactions included, for the different configurations shown in (c). The interaction energy is defined in terms of the adsorption energies, between $\mathrm{C}_{60}$ and $\mathrm{G}$, or $\mathrm{G} / \mathrm{h}-\mathrm{BN}$ heterostructure: $E_{i}=E\left[C_{60}\right]+E[A]-E\left[C_{60} / A\right]$, where $A=G, G / h-B N$. The open bars are for $\mathrm{C}_{60} / \mathrm{G}$, while the solid blue bars are for $\mathrm{C}_{60} / \mathrm{G} / \mathrm{h}-\mathrm{BN}$. 

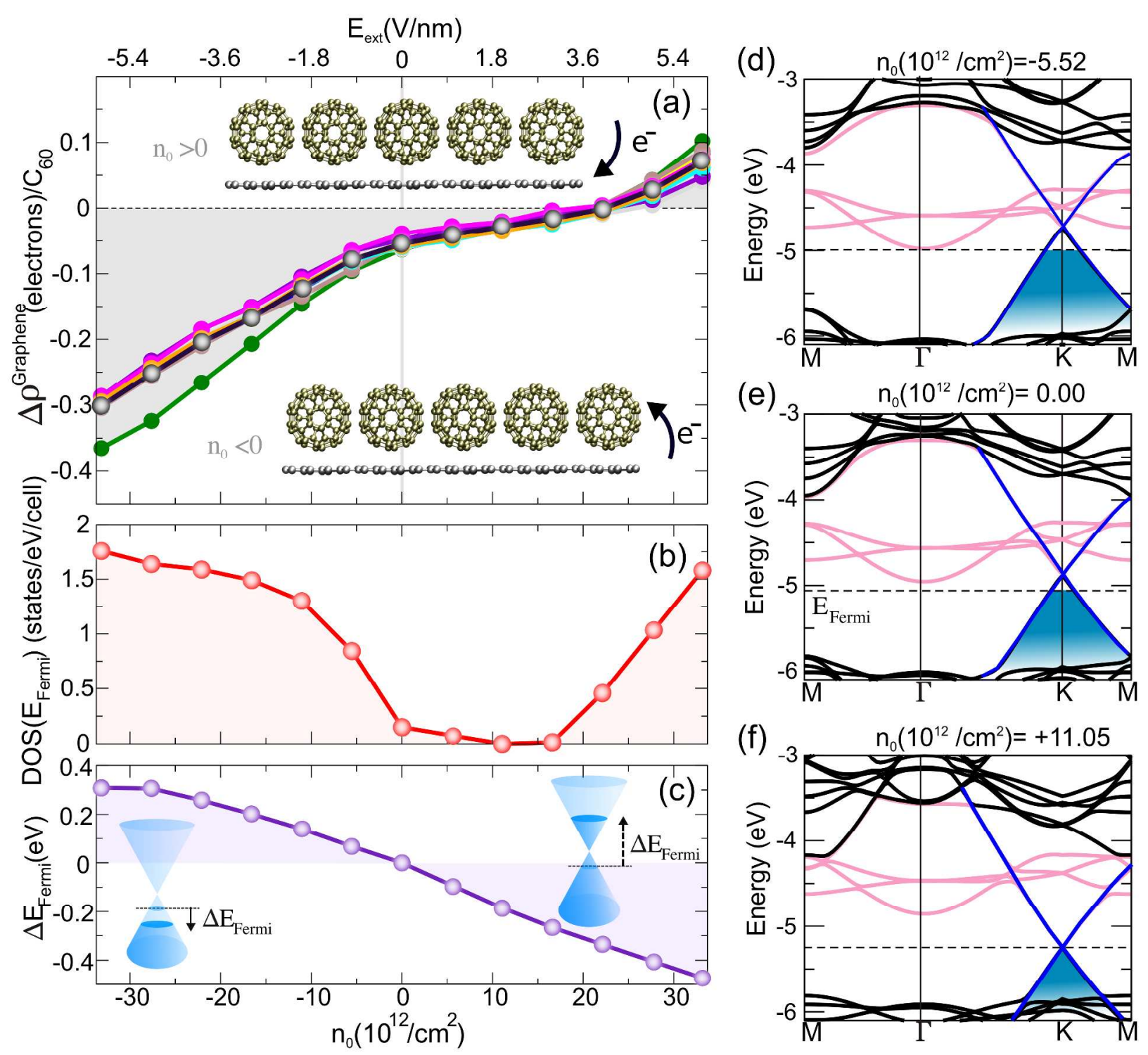

Figure 2. First-principles density functional theory calculations for $C_{60} /$ graphene $\left(C_{60} / G\right)$ interfaces. (a) Calculated charge transfer (in electrons per $\mathrm{C}_{60}$ interfacial molecule) to graphene as a function of the external electric field $\left(\mathrm{E}_{\text {ext }}\right)$ induced-carrier density in graphene $\left(\mathrm{n}_{0}\right)$. Both scales are represented along the $\mathrm{x}$-axis. All nine $\mathrm{C}_{60}$ /graphene geometries presented in Fig. 1(c) are represented, with several data curves superposed on top of each other at the present density scale. The black curve shows the average of all configurations. The green curve (Dimer/Hole) has a slightly larger charge transfer at large values of $n_{0}$. A cut through the data set at $n_{0}=0$, with 
the different configurations identified, is presented in Fig. 3a. Positive $\Delta \rho$ means that electrons are transferred from $\mathrm{C}_{60}$ to graphene, which can occur with sufficient gate bias (i.e. high enough positive $n_{o}$ ). (b) Variation of the density of states at the Fermi level as a function of $n_{0}$ for the Dimer-Hole configuration of $\mathrm{C}_{60} / \mathrm{G}$. Similar results are also observed for the other interface geometries (not shown). (c) Variation of the Fermi level of the combined $\mathrm{C}_{60} / \mathrm{G}$ system as a function of $n_{0}$. Notice that the position of the Fermi level is relative to that at zero bias where a finite charge transfer is already observed. Positive (negative) bias shifts upward (downward) in energy the Fermi level (insets). (d)-(f) Electronic band structures of the $C_{60} / G$ heterostructure with a Dimer/Hole configuration at different gate bias. Graphene states are highlighted in blue and $\mathrm{C}_{60}$ bands in faint red. Fermi level is shown by the dashed-line in each panel. 

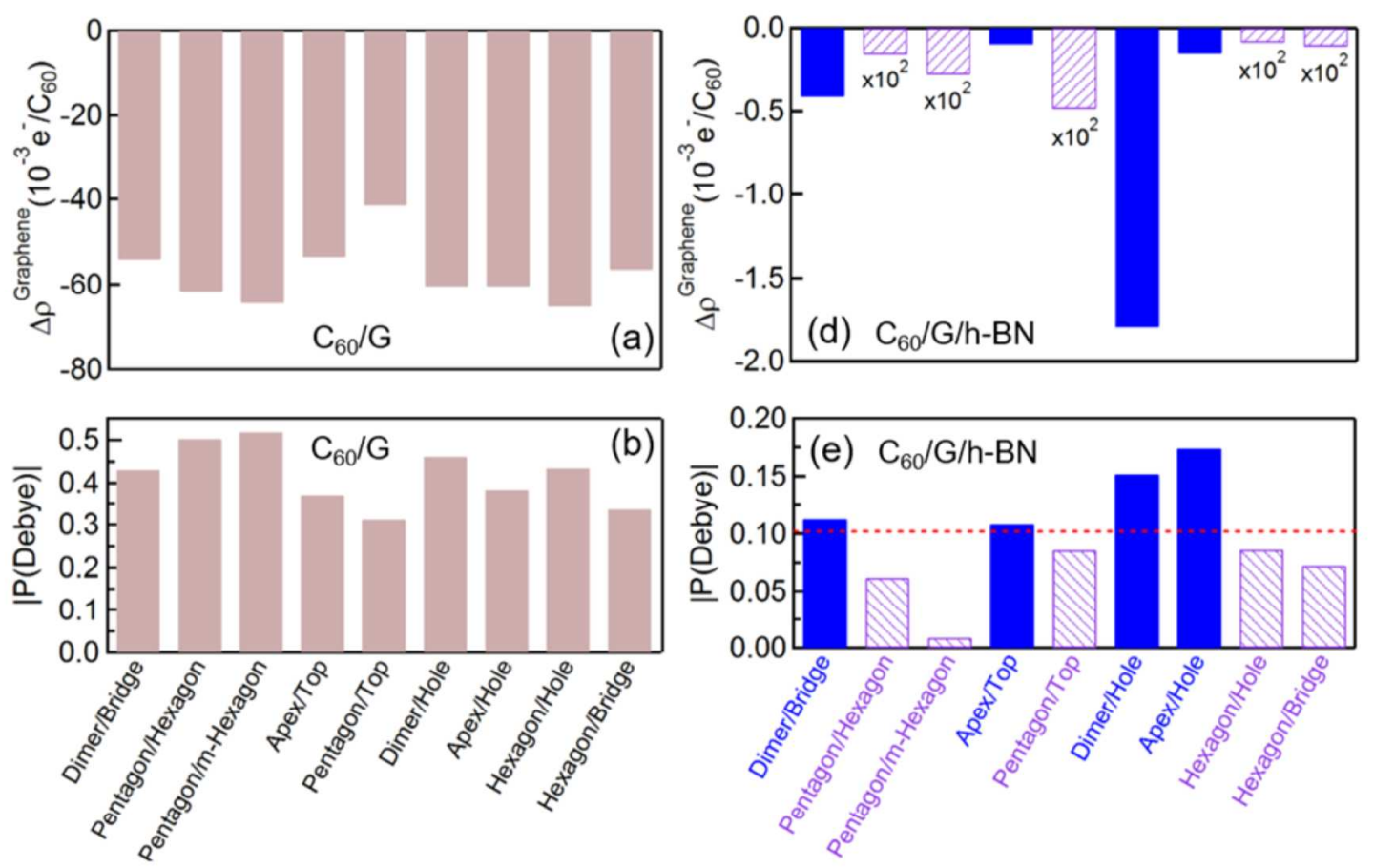

(c)

$\mathrm{C}_{60} / \mathrm{G}$

(f)

$\mathrm{C}_{60} / \mathrm{G} / \mathrm{h}-\mathrm{BN}$
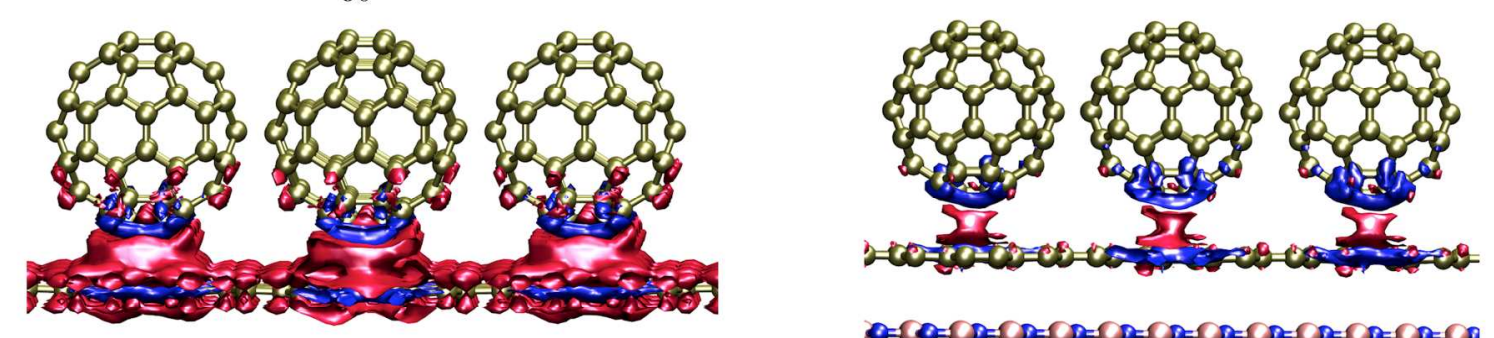

$\angle O=C O=C D=C D=C D=C O=C O=C D=C D=C O=C O=C O=C O$

Figure 3. vdW-DFT calculations for total interfacial charge transfer, induced dipole moment, and charge difference density for the $\mathrm{C}_{60} / \mathrm{G}$ and $\mathrm{C}_{60} / \mathrm{G} / \mathrm{h}-\mathrm{BN}$ systems, all for $\mathrm{n}_{0}=0$. (a) Charge transfer and (b) induced dipole moment for $\mathrm{C}_{60} / \mathrm{G}$. The dipole moment vector points from $\mathrm{C}_{60}$ to graphene. (c) Charge difference density for $\mathrm{C}_{60} / \mathrm{G}$ assuming a Dimer/Hole orientation. (d) Charge transfer and (e) induced dipole moment for $\mathrm{C}_{60} / \mathrm{G} / \mathrm{h}-\mathrm{BN}$. In (d), data for the hatched purple open bars have been amplified by $10^{2}$ for readability. The dashed horizontal red line in (e) delineates an induced dipole threshold of 0.1 Debye. Dipole data above this are plotted in 
solid blue, while data below this are plotted in hatched purple (see text). (f) Charge difference density for $\mathrm{C}_{60} / \mathrm{G} / \mathrm{h}-\mathrm{BN}$ assuming a Dimer/Hole orientation. For (c) and (f), blue and red isosurfaces represent positive and negative charges, respectively. 

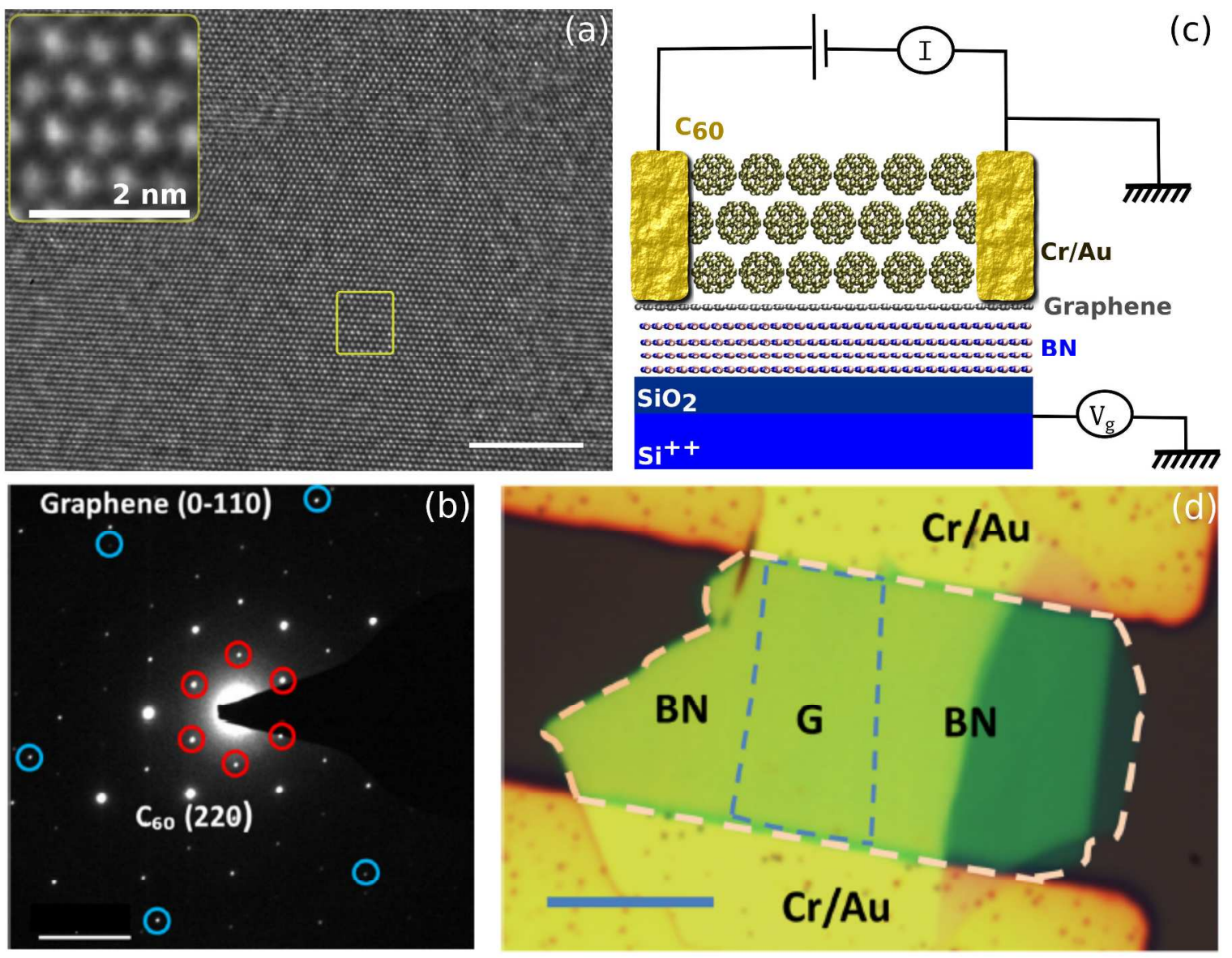

Figure 4. (a) TEM image of a $\sim 15 \mathrm{~nm}$ thick $\mathrm{C}_{60}$ film deposited on suspended graphene under UHV conditions. High crystallinity is clearly observed (scale bar 10nm). The inset is a blow-up of the region outlined by the yellow box, and highlights the close-packed structure of the $\mathrm{C}_{60} \mathrm{~S}$ (bright dots correspond to the projected lattice images of the $\mathrm{C}_{60}$ molecular columns). (b) Electron diffraction for $\mathrm{C}_{60}$ deposited on suspended graphene under UHV conditions.

Graphene (blue circles) and $\mathrm{C}_{60}$ (red circles) diffraction spots are identified (scale bar $2 \mathrm{~nm}^{-1}$ ) (c) Schematic representation of $\mathrm{C}_{60} / \mathrm{G} / \mathrm{h}-\mathrm{BN}$ device used for transport measurements. The device can be electrically probed prior to, and after, $\mathrm{C}_{60}$ deposition. (d) Optical image of a G/h-BN 
heterostructure contacted with $\mathrm{Cr} / \mathrm{Au}$ electrodes prior to $\mathrm{C}_{60}$ deposition. The dotted lines indicate the border of the graphene (blue) and h-BN (pink) flakes (scale bar 20 $\mu \mathrm{m}$ ). 

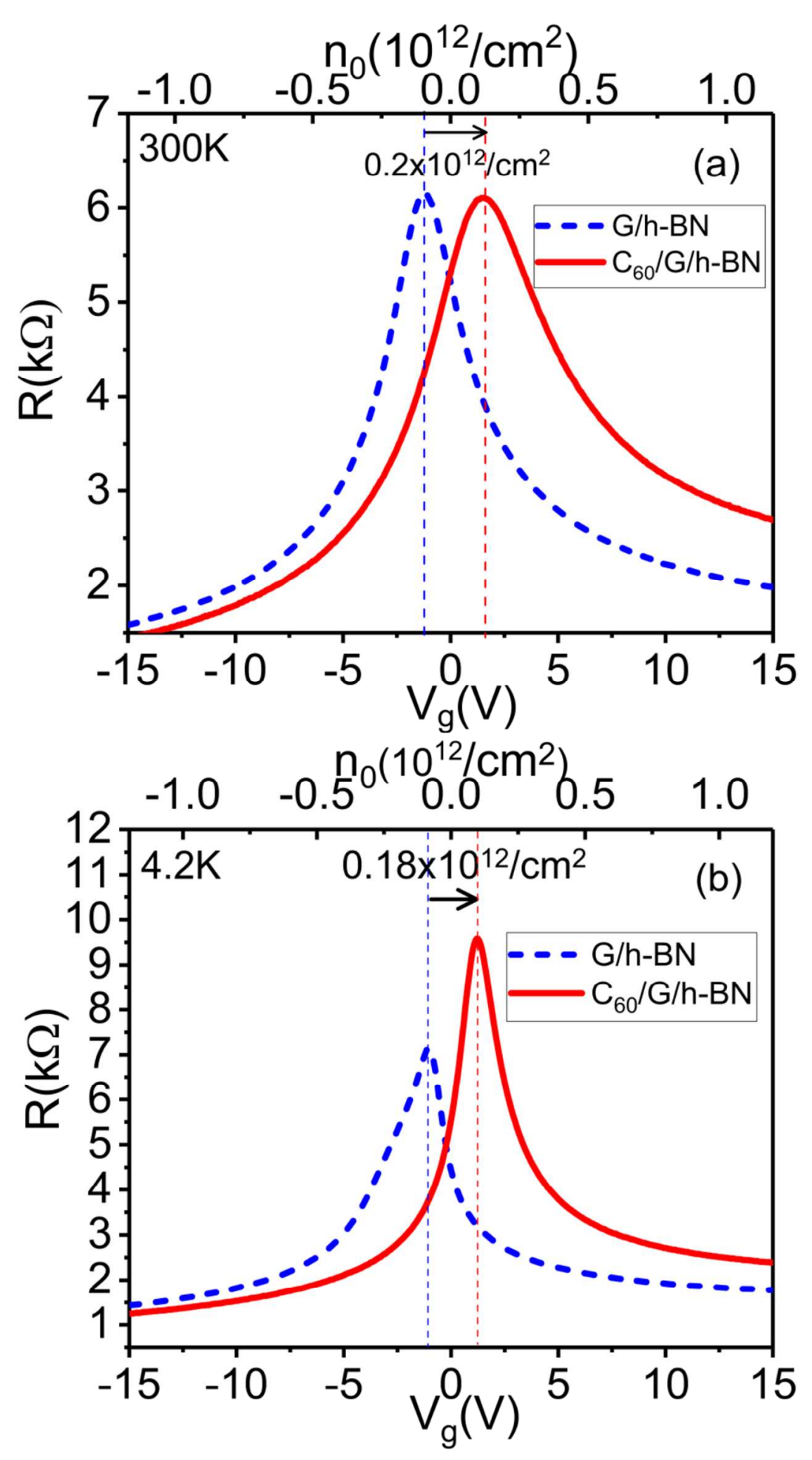

ACS Paragon Plus Environment 
Figure 5. Gate voltage dependence of the resistance of graphene/h-BN devices before (dotted line, blue) and after (solid line, red) deposition of $\approx 15 \mathrm{~nm}$ high crystallinity $\mathrm{C}_{60}$. Deposition conditions are identical to samples in Figure 4. Samples are measured at (a) 300K and (b) $4.2 \mathrm{~K}$. Shift of peak to the right indicates hole doping of the graphene by the $\mathrm{C}_{60}$. Mobilities extracted from gate voltage dependence and presented in Table 1.

\section{For Table of Contents Only}
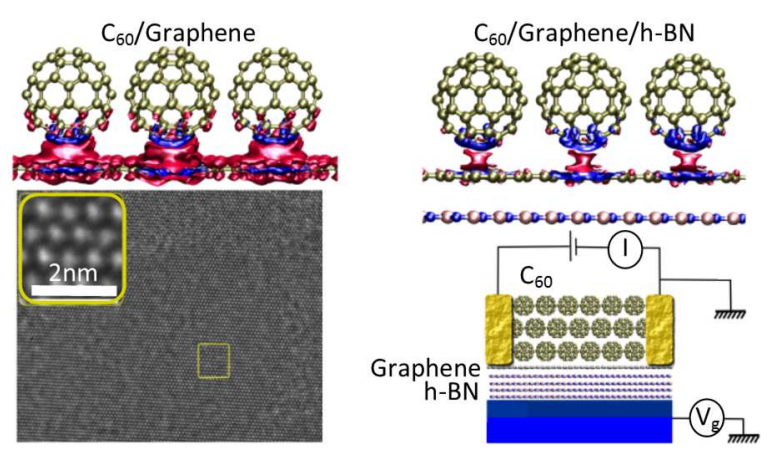